\title{
Influence of combined therapy with conventional and herbal medicines on liver function in 138 inpatients with abnormal liver transaminase levels
}

\author{
Jun Hyuk Shin ${ }^{1}$ Kyuseok Kim² and Hae Jeong $\mathrm{Nam}^{2 *}$ (i)
}

\begin{abstract}
Background: To evaluate the influence of combined therapy of conventional and herbal medicines on liver function.

Methods: This study was a retrospective chart review. A total of 138 patients with abnormal liver transaminase levels at the time of admission were included in this study. We evaluated the influence of combined therapy of conventional and herbal medicines on liver transaminase levels over a period of at least 2 weeks at Kyung Hee University Korean Medical Hospital. Analyses were performed using SPSS version 17.0 for Windows. Paired T-tests were used to examine the significance of differences in AST, ALT, and GGT levels at the time of admission and discharge.
\end{abstract}

Results: We found that combined therapy reduced levels of aspartate aminotransferase (AST), alanine aminotransferase (ALT), and gamma glutamyl transferase (GGT) to a statistically significant level. Specifically, there were $48,66,104$ subjects who exhibited abnormal AST, ALT and GGT levels at admission, which was reduced to 13, 37, and 64 subjects after combined therapy, respectively. Some subjects exhibited worsening levels of liver transaminases after combined therapy, so we used the $x^{2}$ test to analyze the influence of combined therapy with conventional and herbal medicines on liver function according to initial liver transaminase levels. According to this analysis, ALT and GGT levels may be more important than AST levels in estimating the influence of combined therapy on patients with abnormal liver transaminase levels.

Conclusions: Based on this retrospective chart review, combined therapy of conventional and herbal medicines would be considered relatively safe. Thus, if patients have abnormal ALT or GGT levels, caution should be taken when suggesting combined therapy with conventional and herbal medicines.

Keywords: Combined therapy of conventional and herbal medicines, Liver function, Liver transaminases, AST, ALT, GGT

\section{Background}

The use of natural and herbal products for medical purposes has increased in many countries over the past few years. [1, 2] Some Asian countries, including Korea, even consider these natural and herbal products as medicines useful for preventing and treating various diseases.

\footnotetext{
* Correspondence: ophthrl@khu.ac.kr

${ }^{2}$ Department of Ophthalmology, Otorhinolaryngology \& Dermatology of Korean medicine, College of Korean Medicine, Kyung Hee University, \#1 Hoegi-dong, Dongdaemun-gu, Seoul 130-701, Republic of Korea

Full list of author information is available at the end of the article
}

Indeed, many patients taking conventional medicine for diseases such as hypertension, diabetes, and hyperlipidemia also take herbal medicines.

As the interest in herbal medicines and supplements increases more and more, it has become necessary to verify the influence of herbal medicines on liver function. Specifically, concerns about hepatotoxicity caused by herbal medicines have been raised in several papers, but remains controversial. [3-6] Indeed, several other studies [7-10] have reported that herbal medicines are safe and, with respect to liver function, are more helpful 
than harmful. Given the growing prevalence of combined therapies of conventional and herbal medicines, a large-scale retrospective study was performed in Germany. [8] The authors of that study reported that $50.6 \%$ of 1,450 inpatients took herbal medicines at the same time as conventional Western medicine, of which 14 (0.97\%) had drug-induced liver injury, although the prognosis of these patients was fine. Similarly, a study performed in Japan reported that only a small percentage of patients taking combined therapies of conventional and herbal medicines experience drug-induced liver injury due to herbal medicine. $[9,10]$

A drawback of the studies described above is that they were performed using patients with normal liver transaminase levels, focusing only on hepatotoxicity. Thus, the influence of combined therapy of conventional and herbal medicines on liver function itself remains unclear.

To generate primary research with respect to evaluate the safety of combined therapy of conventional and herbal medicines on liver function, we selected hospitalized patients who had more than one abnormal value of aspartate aminotransferase (AST), alanine aminotransferase (ALT), and gamma glutamyl transferase (GGT) at the first examination. We then evaluated the influence of combined therapy ( $>2$ weeks) on changes in AST, ALT and GGT levels.

\section{Methods}

\section{Subjects}

We analyzed patients who were hospitalized in Kyung Hee University Korean Medical Hospital. All patients were selected according to the following inclusion and exclusion criteria.

\section{Inclusion criteria}

(1) Patients who took herbal medicine combined with conventional medicine during hospitalization.

(2) Patients who had more than one abnormal value of AST, ALT, and GGT at first lab finding. AST $>40 \mathrm{U} / \mathrm{L}$, ALT $>40 \mathrm{U} / \mathrm{L}$, and/or GGT $>50 \mathrm{U} / \mathrm{L}$.

\section{Exclusion criteria}

(1) Patients who were lost to follow-up for AST, ALT, and GGT levels before discharge.

\section{Data collection and analysis}

The general characteristics of the study subjects, information about medications, and AST, ALT, and GGT levels at admission and discharge were collected from charts and analyzed retrospectively. Categorical data are presented as 'number (N) (\%)' while continuous-type data are presented as the 'mean \pm standard deviation (SD)'. Analyses were performed using SPSS version 17.0 for Windows. Paired T-tests were used to examine the significance of differences in AST, ALT, and GGT levels at the time of admission and discharge. All differences were considered significant at $P<0.05$.

\section{Ethical issues}

This study was approved by the Institutional Review Board of Kyung Hee University Korean Medical Hospital. (IRB No. KOMCGIRB 2013-135)

\section{Results}

\section{General characteristics of study subjects}

The study consisted of a total of 138 subjects, 82 of them were male and 56 were female. The mean age was $60.12 \pm$ 14.72 years old. The average interval of liver function tests was $41.87 \pm 33.32$ days. The most frequent disease for admission was cerebrovascular disease (ex. cerebral hemorrhage, cerebral infarction, subarachnoid hemorrhage, etc.), followed by the musculo-skeletal disorder, the psychiatric disorder (ex. depression, insomnia, etc.), the peripheral nerve palsy (ex, Bell's palsy, paralytic strabismus, etc.), the vertigo and other diseases (idiopathic sudden hearing loss, stomach pain, post-herpetic neuralgia, and acute hepatitis). The average number of comorbidities was 2.224. The most frequent comorbidity is hypertension, followed by diabetes mellitus, cardiovascular disorders, and hyperlipidemia. Total 58 subjects had hypertension and thirty, twenty-two, and fifteen subjects had these comorbidities respectively. Thirty nine subjects had no comorbidity. The average number of conventional medicines being taken by each patient was 6.25 . The most frequently used conventional medicine comprised anti-hypertension drugs: 126 of total 138 patients were being treated with anti-hypertension drugs (52 calcium channel blockers, $30 \quad \beta$-adrenergic blockers, 29 angiotensin II receptor antagonists, and 15 diuretics). The second and the third frequently used conventional medicine was anticoagulants \& antithrombotics and agents for the digestive system respectively, followed by antiepileptics \& skeletal muscle relaxants, agent for arteriosclerosis, antidepressants \& tranquilizers, antidiabetics, and antiplatelet. It is uncertain for most subjects to identify the cause of liver biochemistry test abnormalities. Only twenty six subjects had non-alcoholic fatty liver disease. Six subjects had chronic hepatitis B and four subjects had alcoholic liver disease. One subject admitted to hospital for acute hepatitis.

Table 1 illustrated clinical characteristics, primary diagnosis for admission, comorbidities, conventional medicines that were taken and cause of liver biochemistry test abnormalities of all subjects.

\section{Comparison of liver transaminase levels before and after combined therapy with conventional and herbal medicines} The mean levels of AST, ALT, and GGT were significantly decreased during hospitalization. Specifically, the average AST level at admission and discharge was 
Table 1 clinical characteristics, primary diagnosis for admission, comorbidities, conventional medicines that were taken and cause of liver biochemistry test abnormalities

\begin{tabular}{|c|c|}
\hline Item & Number (\%) \\
\hline Number of patients & $138(100)$ \\
\hline Gender & Male 82 (59.42), Female $56(40.58)$ \\
\hline Age $($ mean $\pm S D)$ & $60.12 \pm 14.72$ \\
\hline Days of liver function test interval & $41.87 \pm 33.32$ \\
\hline \multicolumn{2}{|l|}{ Primary diagnosis } \\
\hline Cerebrovascular disease & $82(59.42)$ \\
\hline Musculo-skeletal disorders & $17(12.31)$ \\
\hline Psychiatric disorders & $15(10.86)$ \\
\hline Peripheral nerve palsy & $13(9.42)$ \\
\hline Vertigo & $6(4.34)$ \\
\hline Other diseases & $5(3.65)$ \\
\hline \multicolumn{2}{|l|}{ Comorbiditis } \\
\hline Average number of comorbiditis & 2.224 \\
\hline Hypertension & $58(42.02)$ \\
\hline Diabetes mellitus & $30(21.73)$ \\
\hline Cardiovascular disorders & $22(15.94)$ \\
\hline Hyperlipidemia & $15(10.86)$ \\
\hline Others & $12(8.69)$ \\
\hline None & $39(28.26)$ \\
\hline \multicolumn{2}{|l|}{ Conventional medicines } \\
\hline $\begin{array}{l}\text { Average number of medicines being } \\
\text { taken }\end{array}$ & 6.25 \\
\hline Anti-hypertension & $126(91.30)$ \\
\hline Anticoagulants \& Antithrombotics & $122(88.40)$ \\
\hline Agent for digestive system & $109(78.98)$ \\
\hline $\begin{array}{l}\text { Antiepileptics \& Skeletal muscle } \\
\text { relaxants }\end{array}$ & $67(48.55)$ \\
\hline Agent for arteriosclerosis & $66(47.82)$ \\
\hline Antidepressants \& Tranquilizers & $66(47.82)$ \\
\hline Antidiabetics & $44(31.88)$ \\
\hline Antiplatelet & $44(31.88)$ \\
\hline Other CNS drugs & $39(28.26)$ \\
\hline Other circulatory agents & $28(20.28)$ \\
\hline Agents for liver diseases & $22(15.94)$ \\
\hline NSIDS & $21(15.21)$ \\
\hline \multicolumn{2}{|l|}{ Others } \\
\hline \multicolumn{2}{|l|}{$\begin{array}{l}\text { Cause of liver biochemistry test } \\
\text { abnormalities }\end{array}$} \\
\hline Acute Hepatitis & $1(0.72)$ \\
\hline Chronic hepatitis B & $6(4.35)$ \\
\hline Chronic hepatitis C & $0(0)$ \\
\hline Alcoholic liver disease & $4(2.90)$ \\
\hline Non-alcoholic fatty liver disease & $26(18.84)$ \\
\hline Others & 101 (73.19) \\
\hline
\end{tabular}

$45.87 \pm 49.68(\mathrm{U} / \mathrm{L})$ and $27.64 \pm 15.42(\mathrm{U} / \mathrm{L})$, respectively. Likewise, the average ALT at admission and discharge was $54.42 \pm 69.87(\mathrm{U} / \mathrm{L})$ and $33.09 \pm 24.29(\mathrm{U} / \mathrm{L})$, while that of GGT was $97.89 \pm 102.57(\mathrm{U} / \mathrm{L})$ and 63.46 $\pm 52.34(\mathrm{U} / \mathrm{L})$, respectively (Table 2 ).

A total of 48 subjects had abnormal AST at admission while 13 subjects showed abnormal AST after combined therapy. Among the 13 subjects who presented with an abnormal AST, 9 subjects showed higher levels and 4 subjects showed lower AST levels compared to admission after combined therapy. A total of 69 subjects had abnormal ALT at admission and 37 subjects exhibited abnormal ALT after combined therapy. Among the 37 subjects who presented with an abnormal ALT, 18 had a higher ALT level while 19 subjects had a lower ALT level after combined therapy. Lastly, 109 subjects had an abnormal GGT at admission, 64 of which had an abnormal GGT after combined therapy. Among the 64 subjects who continued to have an abnormal GGT, 27 actually had a higher GGT level while 37 had a lower GGT level after combined therapy compared to the time of admission.

From a statistical standpoint, the average AST level at admission was $81.29 \pm 71.75(\mathrm{U} / \mathrm{L})$, which decreased to $34.71 \pm 20.77(\mathrm{U} / \mathrm{L})$ after combined therapy, with a significance probability less than 0.001 . Likewise, the average of admission ALT was $87.85 \pm 89.65$ (U/L), which decreased to $42.18 \pm 25.27$ (U/L) after combined therapy, with a significance probability less than 0.001 . Finally, the average GGT level at admission was $119.33 \pm 109.86$ (U/L), which decreased to $72.72 \pm 55.38$ (U/L) after combined therapy GGT, the significance of which was lower than 0.001 (Table 2).

Considering the period of combined therapy with conventional medicine and herbal medicine, the levels of AST, ALT, and GGT in subjects belonging to the 2-4 week treatment group were significantly decreased $(P=$ 0.003, $P=0.003$, and $P=0.004$, respectively). Likewise, for the 4-8 weeks group, AST and GGT levels were decreased significantly $(P=0.025$ and $P=0.011$, respectively), while the ALT level was not significantly changed. $(P=0.070)$. Similar to the $4-8$ week group, in subjects who received combined therapy for more than 8 weeks, the levels of AST and GGT were significantly decreased ( $P=0.013$ and $P=0.001$, respectively), while that of ALT was not significantly changed $(P=0.110)$ (Table 3$)$.

\section{The influence on AST, ALT, and GGT change after} combined therapy with conventional and herbal medicine Although above results of liver transaminase levels showed safety of combined therapy, some subjects exhibited worsening levels of liver transaminases after combined therapy, so we next used the $x^{2}$ test to analyze the influence of combined therapy with conventional 
Table 2 Comparison of AST, ALT and GGT levels of all subjects and each subject group with abnormal liver transaminase levels before and after combined therapy with conventional and herbal medicines

\begin{tabular}{|c|c|c|c|c|c|}
\hline Comparison & Variables(unit) & $\mathrm{N}$ & $\begin{array}{l}\text { Admission } \\
(\text { Mean } \pm \text { SD) }\end{array}$ & $\begin{array}{l}\text { Discharge } \\
(\text { Mean } \pm S D)\end{array}$ & $P$-Value \\
\hline \multirow[t]{3}{*}{ AST, ALT and GGT levels among all subjects } & AST(U/L) & 138 & $45.87 \pm 49.68$ & $27.64 \pm 15.42$ & $<0.001^{*}$ \\
\hline & $\mathrm{ALT}(\mathrm{U} / \mathrm{L})$ & 138 & $54.42 \pm 69.87$ & $33.09 \pm 24.29$ & $<0.001^{*}$ \\
\hline & GGT(U/L) & 138 & $97.89 \pm 102.57$ & $63.46 \pm 52.34$ & $<0.001^{*}$ \\
\hline \multirow[t]{3}{*}{ AST, ALT and GGT of each subject group with abnormal liver transaminase levels } & $\mathrm{AST}(\mathrm{U} / \mathrm{L})$ & 48 & $81.29 \pm 71.75$ & $34.71 \pm 20.77$ & $<0.001^{*}$ \\
\hline & $\mathrm{ALT}(\mathrm{U} / \mathrm{L})$ & 66 & $87.85 \pm 89.65$ & $42.18 \pm 25.27$ & $<0.001^{*}$ \\
\hline & GGT(U/L) & 104 & $119.33 \pm 109.86$ & $72.72 \pm 55.38$ & $<0.001^{*}$ \\
\hline
\end{tabular}

*Analyzed by paired $T$-test, significant: $P<0.05$

and herbal medicines on liver function according to initial liver transaminase levels. Considering the categorization of the liver transaminases, the prevalence of abnormal ALT and GGT after the combined therapy with conventional and herbal medicines in the abnormal group at baseline was 6.55 and 4.02 times higher than in normal range group at baseline, respectively. ( $P=0.000$ and $P=0.002$, respectively) (Table 4).

\section{Discussion}

A growing number of people are taking conventional medicines for the treatment of diseases like hypertension, hyperlipidemia, and diabetes, which has coincided with an increase in the use of herbal medicines. [1,2] Therefore for safe use of combined therapy with conventional and herbal medicines, it is necessary to study the influence of combine therapy on patients, especially with respect to the liver.

Several previous Korean studies have analyzed the influence of herbal medicine or combined therapy with conventional and herbal medicines on liver function, reporting that herbal medicines can be considered safe for liver function.[11-13] Two other studies [11, 12] were performed using admitted patients similar to the present study; however, they did not distinguish between patients with normal and abnormal liver transaminase levels.
Indeed, most of the study subjects in the aforementioned studies had normal liver transaminases, and no effort made to separate patients who took herbal medicines from those who took combined therapy. A cross-sectional study by Park et al [13] evaluated herbal medicine intake and abnormal liver function. In that study abnormal liver function was not related with history of herbal medicine intake, but was related with male sex and overweight status. Specifically, among a total of 497 patients, 49 had not taken any medicine, 219 patients had taken only conventional medicine, 72 patients had taken only herbal medicine, and 157 patients had taken combined therapy. However, the authors of that study compared only two groups as follows: 219 patients who had taken only conventional medicine and 72 patients who had taken only herbal medicine.

Patients with underlying liver disease have a higher morbidity of liver injury caused by medicines compared with healthy individuals. [14] We assumed that if combined therapy with conventional and herbal medicine is harmful for liver function, patients with abnormal liver transaminase levels could be sensitive to combined therapy. Therefore, we focused on admitted patients at our hospital with abnormal liver transaminase levels to more clearly study the influence of combined therapy on liver

Table 3 Comparison of AST, ALT, and GGT of subjects according to the length of combined therapy with conventional and herbal medicines

\begin{tabular}{|c|c|c|c|c|c|}
\hline Duration & $\mathrm{N}$ & Variables(Unit) & $\begin{array}{l}\text { Admission } \\
(\text { Mean } \pm \text { SD) }\end{array}$ & $\begin{array}{l}\text { Discharge } \\
\text { (Mean } \pm \text { SD) }\end{array}$ & P-Value \\
\hline \multirow[t]{3}{*}{ 2-4 weeks } & 58 & AST(U/L) & $47.55 \pm 56.49$ & $28.72 \pm 18.29$ & $0.003^{*}$ \\
\hline & & $A L T(U / L)$ & $64.86 \pm 79.30$ & $36.29 \pm 26.64$ & $0.003^{*}$ \\
\hline & & GGT(U/L) & $101.79 \pm 117.43$ & $68.22 \pm 54.09$ & $0.004^{*}$ \\
\hline \multirow[t]{3}{*}{ 4-8 weeks } & 52 & AST(U/L) & $39.62 \pm 31.30$ & $28.96 \pm 14.19$ & $0.025^{*}$ \\
\hline & & $\mathrm{ALT}(\mathrm{U} / \mathrm{L})$ & $41.83 \pm 24.62$ & $34.00 \pm 25.60$ & 0.070 \\
\hline & & GGT(U/L) & $93.23 \pm 104.11$ & $65.38 \pm 53.90$ & $0.011^{*}$ \\
\hline \multirow[t]{3}{*}{ More than 8 weeks } & 28 & AST(U/L) & $54.00 \pm 61.53$ & $22.96 \pm 9.57$ & $0.013^{*}$ \\
\hline & & $\mathrm{ALT}(\mathrm{U} / \mathrm{L})$ & $56.18 \pm 99.01$ & $24.79 \pm 12.76$ & 0.110 \\
\hline & & GGT(U/L) & $98.46 \pm 61.04$ & $50.04 \pm 44.64$ & $0.001^{*}$ \\
\hline
\end{tabular}

*Analyzed by paired T-test, significant: $P<0.05$ 
Table 4 The influence on AST, ALT and GGT change following combined therapy with conventional and herbal medicines

\begin{tabular}{|c|c|c|c|c|c|}
\hline \multirow{3}{*}{$\begin{array}{l}\text { Before the combined therapy with conventional and herbal } \\
\text { medicines } N(\%)\end{array}$} & \multicolumn{5}{|c|}{ After the combined therapy with conventional and herbal medicines $\mathrm{N}(\%)$} \\
\hline & & & Normal range & Abnormal range & P-value \\
\hline & \multirow[t]{2}{*}{ AST } & Normal range & $85(61.6 \%)$ & $6(4.3 \%)$ & \multirow[t]{2}{*}{0.132} \\
\hline & & Abnormal range & $40(29.0 \%)$ & $7(5.1 \%)$ & \\
\hline & \multirow[t]{2}{*}{$\mathrm{ALT}$} & Normal range & $65(47.1 \%)$ & $8(5.8 \%)$ & \multirow[t]{2}{*}{$0.000^{*}$} \\
\hline & & Abnormal range & $36(26.1 \%)$ & $29(21.1 \%)$ & \\
\hline & \multirow[t]{2}{*}{ GGT } & Normal range & $27(19.6 \%)$ & $8(5.8 \%)$ & \multirow[t]{2}{*}{$0.002^{*}$} \\
\hline & & Abnormal range & $47(34.1 \%)$ & $56(40.6 \%)$ & \\
\hline
\end{tabular}

${ }^{*}$ Analyzed by $X^{2}$ test, significant: $P<0.05$

function. Conventional medicines, especially those for hypertension, hyperlipidemia, and diabetes mellitus, are typically taken long-term. Likewise, herbal medicines are typically taken long-term rather than short-term because their effect is slow to manifest. Thus, we limited our analysis to patients who had taken combined therapy longer than 2 weeks.

This study didn't attempt to confirm the efficacy of combined therapy but to evaluate the influence of combined therapy on liver function. Usually we clinicians couldn't check every single medicine that has taken by patients, whether it is conventional or herbal. Therefore we roughly checked conventional and herbal medicines.

Herbal medicines are usually composed of several different types of herbs. Thus, we also documented the specific herbs being taken by patients. A total of 202 different kinds of herbs were used among the 138 enrolled patients. The most frequently used herbs were Rhemannia glutinosa (Gaertner) Liboschitz and Glycyrrhiza uralensis Fisch, which were used by all 138 patients. In addition, there were seven herbs used by greater than 100 patients: Poria cocos (Schw.) Wolf, Panax ginseng C. A. Mey. (= Panax schinseng Nees), Atractylodes macrocepha-la Koidz, Angelica gigas Nakai, Scutellaria baicalensis Georgi, Paeonia albiflora Pallas var. trichocarpa Bunge, and Zingiber officinale Rosc. Finally, 90 of the 202 different herbs were used by less than 10 patients, and only 26 herbs were used by more than half of patients.

There are several herbs which were considered harmful for liver function. In Korea, Yun et al [7] and Park et al [15] reported that there some herbs, for example Ephedra sinica Stapf, Scutellaria baicalensis Georgi, Dictamnus dasycarpus Turcz., Aconitum carmichaeli Debx (= Aconitum chinense Sieb. et Zucc.), Euphorbia kansui Liou ex Wang, Croton tiglium Linne, Pinellia ternata (Thunb.) Breit., Rheum palmatum L., Sinomenium acutum Rehder et Wils., Polygonum multiflorum Thunb., Valeriana fauriei Briquet could be harmful for liver function. F. Stickel \& D. Shouval [3], C. Korth [4], and E.S. Bjornsson [16] also reported about the herbs which could be potentially harmful for liver function. Some of the herbs in these studies, for example Agastache rugosa (Fisch. et Meyer) O. Kuntze,
Tussilago farfara L. [3], Chelidonium majus L., Cimicifuga heracleifolia Kom. [4], Plantago asiatica L. [16] were used but the other herbs were not used for the subjects in this study.

Table 5 illustrated the herbs which could be harmful for liver function were used in this study.

The present study represents a primary research study on the safety of combined therapy with respect to liver function. Despite the positive finding that the average AST, ALT, and GGT levels of the 138 subjects were significantly reduced, there were a few subjects who had abnormal liver transaminase levels after combined therapy. Thus, in order to confirm the main factors by which liver transaminases predict the influence of combined therapy on liver function, which will be useful for

Table 5 The number of cases prescribed herbs previously reported as hepatotoxic agents

\begin{tabular}{lll}
\hline Herbs & Commonly used for & $\begin{array}{l}\text { Number of } \\
\text { cases }\end{array}$ \\
\hline Glycyrrhiza uralensis Fisch. & $\begin{array}{l}\text { Essential herbs for } \\
\text { herbal medicine }\end{array}$ & 138 \\
Scutellaria baicalensis Georgi & Fever & 104 \\
Pinellia ternata (Thunb.) Breit. & Vertigo, Nausea & 84 \\
Rheum palmatum L. & Constipation & 60 \\
Cimicifuga heracleifolia Kom. & Menopausal syndrome & 52 \\
Coptis deltoidea C.Y. Cheng & Fever & 48 \\
et Hsiao & & \\
Ephedra sinica Stapf. & Edema, Obesity & 39 \\
Agastache rugosa (Fisch. et Meyer) & Common cold, Blood & 39 \\
O. Kuntze & circulation & \\
Plantago asiatica L. & Constipation & 30 \\
Aconitum carmichaeli Debx & Heart stimulant & 19 \\
Aconitum ciliare DC. & Blood circulation & 7 \\
Tussilago farfara L. & Cough, Common cold & 7 \\
Sinomenium acutum & Edema & 6 \\
Rehder et Wils. & & 6 \\
Corydalis ternata Nakai & For extravasated blood & 6 \\
Polygonum multiflorum Thunb. & Adaptogen & 4 \\
Chelidonium majus L. & Fever & 1 \\
\hline
\end{tabular}


clinicians who are considering suggesting combined therapy with conventional and herbal medicines, we analyzed subjects with abnormal liver transaminases using the $x^{2}$ test. According to this analysis, ALT and GGT levels may be more important than AST levels in estimating the influence of combined therapy on patients with abnormal liver transaminase levels. Thus, if patients have abnormal ALT or GGT levels, caution should be taken when suggesting combined therapy with conventional and herbal medicines.

This study had some weak points. First, the subjects in this study consisted of individuals who were admitted to the hospital, and thus were able to eat a healthy diet, did not consume alcoholic drinks, and were able to rest. Under such circumstances it is likely that the liver may be better able to repair itself. Therefore, future studies should be conducted in an outpatient setting. Secondly, unlike conventional medicines, herbal medicines consist of natural herbs, which can vary significantly with respect to quality. Thus, herbal medicines made with cheap herbs contaminated by agricultural chemicals or heavy metals may be harmful to liver compared with products of a higher quality. Thirdly, this study was a primary retrospective research study. In order to more fully evaluate the influence of combine therapy on liver function, it will be necessary to perform a randomized control study. Finally, this finding is a short term observation and that data regarding long term outcomes would be necessary to reach more definitive conclusions.

\section{Conclusions}

The results of this retrospective study suggest that combined therapy with conventional and herbal medicines is relatively safe on liver function. However, some patients had increased liver transaminase levels, highlighting the importance of routine lab examination to continuously monitor patients with abnormal liver transaminase levels.

\section{Abbreviations}

AST: Aspartate aminotransferase; ALT: Alanine aminotransferase; GGT: Gamma glutamyl transferase

\section{Acknowledgements}

Not applicable.

\section{Funding}

Not applicable.

\section{Availability of data and materials}

The datasets generated and analyzed during the current study are not publicly available to maintain patient confidentiality but are available from the corresponding author on reasonable request.

\section{Authors' contributions}

HJN conceived of the study. JHS and HJN conducted the study. KSK performed the statistical analysis. JHS and HJN led the writing of the manuscript. HJN and KSK commented on the analytic plan and interpretation. All authors

contributed to editing and final approval of manuscript.

\section{Competing interests}

The authors declare that they have no competing interests.

\section{Consent for publication}

Not applicable.

Ethics approval and consent to participate

This study was reviewed and approved by the Institutional Review Board of Kyung Hee University Korean Medical Hospital. (IRB No. KOMCGIRB 2013-135). Written informed consent was obtained from the participants.

\section{Author details}

${ }^{1}$ Graduate school of Korean medicine, Kyung Hee University, \#1 Hoegi-dong, Dongdaemun-gu, Seoul 130-701, Republic of Korea. ${ }^{2}$ Department of Ophthalmology, Otorhinolaryngology \& Dermatology of Korean medicine, College of Korean Medicine, Kyung Hee University, \#1 Hoegi-dong, Dongdaemun-gu, Seoul 130-701, Republic of Korea.

Received: 4 September 2015 Accepted: 14 November 2016

Published online: 01 December 2016

\section{References}

1. Eisenberg DM, Davis RB, Ettner SL, Appel S, Wilkey S, Van Rompay M, et al Trends in alternative medicine use in the United States, 1990-1997: results of a follow-up national study. JAMA. 1998;280:1569-75.

2. Posadzki $P$, Watson LK, Alotaibi A, Ernst E. Prevalence of use of complementary and alternative medicine(CAM) by patients/consumers in the UK: systemic review of surveys. Clin Med. 2013;13:126-31.

3. Stickel F, Shouval D. Hepatotoxicity of herbal and dietary supplements: an update. Arch Toxicol. 2015;89:851-65.

4. Korth C. Drug-induced hepatotoxicity of select herbal therapy. J Pharm Pract. 2014;27(6):567-72.

5. Kang SH, Kim Jl, Jeong KH, Ko KH, Ko PG, Hwang SW, et al. Clinical characteristics of 159 cases of acute toxic hepatitis. Korean J Hepatol. 2008;14(4):483-92.

6. Lee WY, Park BC, Kim HS, Joung KO, Yoon SI, Kwon SK, et al. A case of acute interstitial nephropathy and toxic hepatitis after short-term herbal medication. Korean J Med. 2007;73(1):103-6.

7. Yun YJ, Park JH, Paeck EK, Park JH, Jeong SK, Park HM, et al. Safety of prescribed Korean herbal medicine on liver function : prospect pilot study. Kor J Ori Physiol Pathol. 2009;23(3):715-22.

8. Melchart D, Linde K, Hager S, Kaesmayr J, Shaw D, Bauer R, et al. Monitoring of liver enzymes in patients treated with traditional Chinese drugs. Complement Ther Med. 1999;7(4):208-16.

9. Mantani N, Kogure T, Sakai S, Goto H, Shibahara N, Kita T, et al. Incidence and clinical features of liver injury related to Kampo(Japanese herbal) medicine in 2,496 cases between 1979 and 1999: Problems of the lymphocyte transformation test as a diagnostic method. Phytomedicine. 2002;9(4):280-7.

10. Mitsuma T. Adverse reactions encountered during treatment with Japanese oriental (Kampo) medicines. Korean J Orient Intern Med. 2002;2:9-14.

11. Kim DM, Kim HK, Cho SY, Kim YS, Naml SS. Retrospective observation of liver function parameters for 101 patients using herbal drugs for one month. J Korean Oriental Med. 2010;31(2):149-57.

12. Rhee SH, Park JS, Yeo HS, Choi YK, Jun CY, Park CH, et al. Relationship between herb-medicine and liver damage. Korean J Oriental Int Med. 2006;27(3):573-80.

13. Park HM, Shin HT, ChS P, Lee SD. A cross-section study between herbal medicine intake and abnormal liver function test results. Kor J Orient Prev Med Soc. 2008;12(2):61-72.

14. Ahn BM. Herbal preparation-induced liver injury. Korean J Gastroenterol. 2004:44(3):113-25

15. Park YC, Park HM, Lee SD. Inducible Mechanisms for Hepatotoxicity caused by Traditional Korean Medicines in a view of Toxicology. J Korean Oriental Med. 2011;32(4):48-67.

16. Bjornsson ES. Drug-induced liver injury: an overview over the most critical compounds. Arch Toxicol. 2015;89:327-34. 